Bangladesh J. Plant Taxon. 23(2): 237-246, 2016 (December)

(C) 2016 Bangladesh Association of Plant Taxonomists

\title{
PHYLOGENY OF GALIUM L. (RUBIACEAE) FROM KOREA AND JAPAN BASED ON CHLOROPLAST DNA SEQUENCE
}

\author{
Keum Seon Jeong, Jae Kwon Shin ${ }^{1}$, Masayuki Maki ${ }^{2}$ and Jae-Hong PaK ${ }^{3}$ \\ Division of Forest Biodiversity, Korea National Arboretum, Pocheon, \\ Gyeonggi-do 487-821, Korea
}

Keywords: Chloroplast DNA; Korean-Japan Galium; Molecular data; Phylogeny.

\begin{abstract}
The present paper deals with the phylogeny and inter-and intragenic relationships using four chloroplast DNA sequences within 19 Galium L. species from Korea and Japan. Maximum parsimony and Bayesian analyses were conducted to clarify the relationships among the section and species. The strict consensus tree had three main clades. Clade I comprises of the only individuals of $G$. paradoxum Maximowicz (sect. Cymogalia), which is distinguished by opposite leaves in the genus, supported by the $100 \%$ bootstrap value (PP: 0.98); Clade II consists of members of eight sections (sect. Galium, sect. Hylaea, sect. Kolgyda, sect. Trachygalium, sect. Leptogalium, sect. Orientigalium, sect. Aparine, and sect. Leiogalium); Clade III comprises members of eight sections (sect. Baccogalium, sect. Lophogalium, sect. Platygalium, sect. Relbunium, sect. Depauperata, sect. Aparinoides, sect. Leiogalium and Trachygalium). The sect. Leptogalium which includes two taxa namely $G$. tokyoense Makino and $G$. dahuricum var. lasiocarpum (Makino) Nakai is paraphyletic. Four taxa of Trachygalium group ( $G$. trachyspermum A. Gray, G. gracilens (A. Gray) Makino, G. pogonanthum Franch. \& Sav., G. koreanum Nakai) were placed from sect. Cymogalia to sect. Platygalium based on molecular and morphological data.
\end{abstract}

\section{Introduction}

Galium L., the largest genus of the tribe Rubieae in the family Rubiaceae (Robbrecht and Manen, 2006), is taxonomically diverse and comprises over 650 species (Govaerts, 2006). Galium is divided into 16 sections based on characters of leaf and fruit by Ehrendorfer et al. (2005). The species of Galium are distributed centrally in temperate regions and are mostly annual and perennial herbaceous plants. The genus is characterized by more than two leaf-like whorls, number of divided petal, rudimentary calyx and a two locular ovary.

Phylogenetic relationships among species of tribe Rubieae including eleven genera have been studied by many researchers (Ehrendorfer et al., 1994, 2014; Manen et al., 1994; Manen and Natali, 1995; Natali et al., 1995, 1996; Soza and Olmstead, 2010). Molecular phylogenetic studies using chloroplast DNA atpB-rbcL intergenic region have shown monophyly of the tribe Rubieae with seven major clades, and confirmed that genera Asperula and Galium is not a monophyletic group (Manen et al., 1994; Natali et al., 1995, 1996). Soza and Olmstead (2010) conducted more clearly molecular phylogenetic analysis of tribe Rubieae using three chloroplast DNA makers and their results indicated that Galium is polyphyletic, and species of Galium occur in three major clades (Clades III, V, VII). Recently, phylogenetic relationships study of tribe Rubieae including

${ }^{1}$ Division of Forest Resource Conservation, Korea National Arboretum, Pocheon, Gyeonggi-do 487-821, Korea.

${ }^{2}$ Division of Ecology and Evolutionary Biology, Graduate School of Life Sciences, Tohoku University, Aoba, Sendai 980-8578, Japan.

${ }^{3}$ Research Institute for Dok-do and Ulleung-do Island, Kyungpook National University, Daegu 702-701, Korea. Corresponding author. Email: jhpak@knu.ac.kr 
some Galium species by Ehrendorfer et al. (2014) has evaluated that genus Galium is paraphyletic. Although there have been several phylogenetic study to investigate relationships of tribe Rubieae, very little is known about phylogenetic relationships among Korean species of Galium. Soza and Olmstead (2010) determined the phylogenetic relationships among Rubieae including members of Galium but this study included only three common species distributed in Korea and Japan. In Korea, twenty taxa of seven sections are currently recognized (Lee, 1995; Lee, 1979; Lee, 2004). G. koreanum Nakai, G. verum var. asiaticum for. pusillum (Nakai) M. Park are endemic to Korea and latter species is restrictedly distributed in Mt. Halla of Jeju Island. G. kikumugura Ohwi is broadly expanded to Japan. Jeong and Pak $(2009,2012)$ conducted morphological and somatic chromosome number counts of Korean Galium. These studies however, provided very little phylogenetic relationships among the species. Therefore, further studies are needed to understand their phylogenetic relationships among Korean Galium species and taxonomic position of Korean and Japan taxa within the Galium spp. occurring worldwide. This study aims to clarify inter-and intragenic relationships within Korean and 10 Japanese Galium species, and to determine the taxonomic position of Korean endemic taxa within the closely related Galium spp. using the chloroplast DNA sequences.

\section{Materials and Methods}

\section{Plant materials}

Total 19 species of Galium distributed in Korea and Japan were collected (Table 1). We selected two outgroup taxa [ Didymaea alsinoides (Cham. and Schltdl.) Standl., and Rubia cordifolia L.] based on the results of the analyses of Soza and Olmstead (2010). The sequences of Galium and outgroups obtained from National Center for Biotechnology Information (NCBI) database with the exception of sequences of sample from Korea-Japan. All sources and voucher specimens of materials were deposited at the Herbarium of Kyungpook National University (KNU).

\section{DNA extraction, amplification and sequencing}

Total genomic DNA was extracted from fresh leaf tissues and field-collected silica-gel dries tissue using the $2 \%$ hexa decyltrimethyl ammonium bromide (CTAB) procedure (Doyle and Doyle, 1987). We amplified the rpoB-trnC region and $\operatorname{trnC}-y c f 6$ region with primers designed by Demesure et al. (1995). The trnL-trnF-ndhJ region was amplified using primers published in Taberlet et al. (1991) and Shaw et al. (2007) (Table 2). Polymerase chain reaction (PCR) conditions were an initial denaturation of $94^{\circ} \mathrm{C}$ for $5 \mathrm{~min}, 35$ cycles of $94^{\circ} \mathrm{C}$ denaturation for $30 \mathrm{~s}$, $48^{\circ} \mathrm{C}-57^{\circ} \mathrm{C}$ annealing for $30 \mathrm{~s}$ extension for $1 \mathrm{~m}$, and final extension at $72^{\circ} \mathrm{C}$ for $10 \mathrm{~min}$. PCR products were purified using the QIAquick PCR purification kit following the instructions of the manufacturer. Sequencing reactions were carried out for the purified PCR products using Big Dye Terminator Cycle Sequencing reagents (Applied Biosystem, Foster city, CA, USA). For sequencing, we used the same primers as those used for PCR. All sequences have been deposited in GenBank (Table 1).

\section{Data analysis}

The DNA sequences were aligned with Clustal X (Thompson et al., 1997). All chloroplast regions were combined and analyzed using Maximum Parsimony (MP) and the Bayesian analyses. Gaps introduced from the alignment were treated as missing characters in subsequent analyses. MP analyses were conducted in a PAUP* (version 4.0b 10; Swofford, 2003) using a heuristic searches with TBR branch swapping and MULTREES option. Relative support of various monophyletic groups revealed in the most parsimonious trees was examined with the bootstrap 
Table 1. Sampling sites of plant materials used for phylogenetic analyses.

\begin{tabular}{|c|c|c|c|c|c|c|}
\hline \multirow{2}{*}{ Taxon } & \multirow{2}{*}{ Locality } & \multirow{2}{*}{ Voucher } & \multicolumn{3}{|c|}{ GenBamk acc. No. } & \multirow[b]{2}{*}{ rpoB-trnC } \\
\hline & & & trnC-ycf6 & $t r n \mathrm{~F}-n d h \mathrm{~J}$ & $\operatorname{Trn} \mathrm{L}$ & \\
\hline \multicolumn{7}{|l|}{$\overline{\text { Sect. Aparine }}$} \\
\hline $\begin{array}{l}\text { Galium spurium var. } \\
\text { echinospermon }\end{array}$ & Chilgok-gun, Korea & $\mathrm{J} 20050310$ & KC339150 & KC339020 & KC339085 & LC062539 \\
\hline \multicolumn{7}{|l|}{ Sect. Aparinoides } \\
\hline \multirow[t]{2}{*}{ G. trifidum } & Jeju-si, Korea & J20060807 & KC339148 & KC339018 & KC339083 & LC062537 \\
\hline & Tokyo metro, Japan & M20100501 & KC339149 & KC339019 & KC339084 & LC062538 \\
\hline \multicolumn{7}{|l|}{ Sect. Cymogalia } \\
\hline \multirow[t]{3}{*}{ G. paradoxum } & Pyeongchang-gun, Korea & J20090814 & KC339164 & KC339034 & KC339099 & LC062552 \\
\hline & Jeongseon-gun, Korea & J20050618 & KC339163 & KC339033 & KC339098 & LC062551 \\
\hline & Muju-gun, Korea & J20100844 & KC339162 & KC339032 & KC339097 & LC062550 \\
\hline \multicolumn{7}{|l|}{ Sect. Hylaea } \\
\hline \multirow[t]{4}{*}{ G. trifloriforme } & Ulleung-gun, Nari, Korea & J20080621 & KC339204 & KC339074 & KC339139 & LC062581 \\
\hline & Ulleung-gun, Korea & $\mathrm{J} 20080635$ & KC339203 & KC339073 & KC339138 & LC062580 \\
\hline & Ulleung-gun, Taehwa, Korea & $\mathrm{J} 20080603$ & KC339205 & KC339075 & KC339140 & LC062582 \\
\hline & Miyagi, Japan & J20100748 & KC339206 & KC339076 & KC339141 & LC062583 \\
\hline \multirow[t]{6}{*}{ G. japonicum } & Ulleung-gun, Nari, Korea & $\mathrm{J} 20080611$ & KC339207 & KC339077 & KC339142 & LC062584 \\
\hline & Ulleung-gun, Nari, Korea & $\mathrm{J} 20080612$ & KC339151 & KC339021 & KC339086 & LC062540 \\
\hline & Jeongeup-si, Korea & $\mathrm{J} 20100845$ & KC339209 & KC339079 & KC339144 & LC062585 \\
\hline & Jeju-si, Korea & $\mathrm{J} 20070901$ & KC 339210 & KC339080 & KC339145 & LC062586 \\
\hline & Miyagi, Japan & $\mathrm{J} 20100758$ & KC339211 & KC339081 & KC339146 & LC062587 \\
\hline & Yamagata, Japan & $\mathrm{J} 20100759$ & KC339212 & KC339082 & KC339147 & LC062588 \\
\hline \multicolumn{7}{|l|}{ Sect. Leptogalium } \\
\hline \multirow{6}{*}{$\begin{array}{l}\text { G. dahuricum var. } \\
\text { lasiocarpum }\end{array}$} & Namyangju-si, Korea & J20100897 & KC339189 & KC339059 & KC339124 & LC062569 \\
\hline & Pyeongchang-gun, Korea & J20090807 & KC339192 & KC339062 & KC339127 & LC062571 \\
\hline & Yeongwol-gun, Korea & J20080926 & KC339155 & KC339025 & KC339090 & LC062543 \\
\hline & Seongju-si, Korea & $\mathrm{J} 20100658$ & KC339188 & KC339058 & KC339123 & LC062568 \\
\hline & Jecheon-si, Korea & J20091021 & KC339190 & KC339060 & KC339125 & LC062570 \\
\hline & Yamagata, Japan & $\mathrm{J} 20100708$ & KC339194 & KC339064 & KC339129 & LC062573 \\
\hline G. kikumugura & Mt. Zao, Japan & $\mathrm{J} 20100765$ & KC339200 & KC339070 & KC339135 & LC062577 \\
\hline G. pseudoasprellum & Miyagi, Japan & J20100789 & KC339202 & KC339072 & KC339137 & LC062579 \\
\hline \multirow[t]{3}{*}{ G. tokyoense } & Pocheon-si, Korea & J20070938 & KC339195 & KC339065 & KC339130 & LC062572 \\
\hline & Pocheon-si, Korea & $\mathrm{J} 20090808$ & KC339193 & KC339063 & KC339128 & LC062574 \\
\hline & Tokyo metro, Japan. & M20090503 & KC339197 & KC339067 & KC339132 & LC062575 \\
\hline \multicolumn{7}{|l|}{ Sect. Platygalium } \\
\hline G. boreale & Yeongwol-gun, Korea & J20050625 & KC339152 & KC339022 & KC339087 & LC062541 \\
\hline & Mongolia & L20090830 & KC339153 & KC339023 & KC339088 & LC062542 \\
\hline G. gracilens & Sunchen-si, Korea & J20090801 & KC339181 & KC339051 & KC339116 & LC062566 \\
\hline & Hwasun-gun, Korea & J20090830 & KC339180 & KC339050 & KC339115 & LC062565 \\
\hline $\begin{array}{l}\text { G. kamtschaticum } \\
\text { var. yakusimense }\end{array}$ & Jeju-si, Korea & J20070907 & KC339166 & KC339036 & KC339101 & LC062553 \\
\hline G. koreanum & Sancheong-gun, Korea & J20100808 & KC339186 & KC339056 & KC339121 & LC062567 \\
\hline G. kinuta & Yeongwol-gun, Korea & J20050626 & KC339167 & KC339037 & KC339102 & LC062554 \\
\hline G. pogonanthum & Hamyang-gun, Korea & J20090504 & KC339172 & KC339042 & KC339107 & LC062559 \\
\hline & Jeju-si, Korea & J20050706 & KC339171 & KC339041 & KC339106 & LC062558 \\
\hline G. trachyspermum & Inje-gun, Korea & J20080906 & KC339170 & KC339040 & KC339105 & LC062546 \\
\hline & Andong-si, Korea & $\mathrm{J} 20070751$ & KC339157 & KC339027 & KC339092 & LC062545 \\
\hline & Gyeongju-si, Korea & $\mathrm{J} 20100913$ & KC339159 & KC339029 & KC339094 & LC062547 \\
\hline & Geoje-si, Korea & $\mathrm{J} 20090327$ & KC339156 & KC339026 & KC339091 & LC062544 \\
\hline & Miyagi, Japan & $\mathrm{J} 20100723$ & KC339160 & KC339030 & KC339095 & LC062548 \\
\hline & Yamagata, Japan & J20100747 & KC339161 & KC339031 & KC339096 & LC062549 \\
\hline Sect. Galium & & & & & & \\
\hline G. verum var. asiaticum & Geoje-si, Korea & J20100524 & KC339173 & KC339043 & KC339108 & LC062563 \\
\hline & Jeju-si, Korea & $\mathrm{J} 20090685$ & KC339174 & KC339044 & KC339109 & LC062562 \\
\hline & Fukui, Japan & M20100503 & KC339176 & KC339046 & KC339111 & LC062561 \\
\hline G. verum var. & Ulsan metro., Korea & $\mathrm{J} 20050830$ & KC339198 & KC339068 & KC339133 & LC062576 \\
\hline $\begin{array}{l}\text { trachycarpum } \\
\text { f. nikkoense }\end{array}$ & Tokushima, Japan & $\mathrm{J} 20100732$ & KC339177 & KC339047 & KC339112 & LC062564 \\
\hline $\begin{array}{l}\text { G. verum var. asiaticum } \\
\text { f. pusillum }\end{array}$ & Jeju-si, Korea & $\mathrm{J} 20050807$ & KC339175 & KC339045 & KC339110 & LC062560 \\
\hline
\end{tabular}


method (Felsenstein, 1985). Bootstrap values were calculated from 1,000 replicates with the random addition and heuristic search options. The Bayesian phylogenetic analyses were conducted with MrBayesver 3.1.2 (Ronquist and Huelsenbeck, 2003). The suitable model was determined to be GTR+I+G for combined sequence data by MrModeltest 2.3 (Nylander, 2004). Each Morkov chain was started from a random tree and run for 1,000,000 generations, sampling a tree every 100 generations. Burn-in time was estimated from the plot of likelihoods generated using the 'sump' command in MrBayes. Posterior probabilities (pp) were based on analysis of post-burn-in tree. Nodes were considered highly supported when pp values were higher than 0.95 (Felesenstein, 1985).

\section{Results and Discussion}

Sequence characteristics

The total of 4,341 lengths of the aligned sequences was used for phylogenetic analysis. Of a total of investigated character sites, 2,793 characters were constant and 824 characters were parsimony informative including out groups. The parsimony analyses generated 10,620most parsimonious trees with a total length of 2,970 steps, a consistency index of 0.65 and a retention index of 0.88.The MP tree with bootstrap values(BP) and PP are shown in Fig. 1.

\section{Phylogenetic analyses}

The strict consensus tree had three main clades (clade I, clade II and clade III). Clade IV is highly supported by the $100 \%$ bootstrap value (PP: 0.98 ) and was sister to the rest of the species, which were grouped in two other clades. This clade was only composed of the individuals of $G$. paradoxum Maxim. Clade IIa is supported $99 \%$ bootstrap value $(\mathrm{PP}<0.95)$. Clade IIb consists of two highly supported subclades (subclade IIa and IIb). Subclade IIa included three taxa: $G$. dahuricum var. lasiocarpum (Makino) Nakai., G. pseudoasprellum Makino and G. triflorum Michx. comprising of Group B. G. triflorum (sect. Trachygalium) was sister to G. dahuricum var. lasiocarpum from Korea-Japan and G. pseudoasprellum from Japan (99\% bootstrap value). Subclade IIb is supported by $91 \%$ bootstrap value $(\mathrm{PP}<0.95)$. This subclade contained 8 taxa from Korea-Japan. It was further divided into Group C and D. Group C contained members of three sections (sect. Galium, sect. Leiogalium and sect. Leptogalium) which are identified by Soza and Olmstead (2010), G. tokyoense Makino, G. kikumugura, and three species belonging to sect. Galium from Korea-Japan. But the G. verum group from Korean and Japanese were not well resolved. In the Group D, G. japonicum (Maxim.) Makino \& Nakai from Korea and Japan is monophyletic, although the individuals of G. trifloriforme Kom. did not form monophyletic group. These two taxa share its most recent common ancestor with G. spurium var. echinospermum (Wallr.) Hayekand G. odoratum (L.) Scop (61\% bootstrap value (PP: 0.96)). Clade III is supported by $91 \%$ bootstrap value (PP: 0.97), comprising eight sections; sect .Baccogalium, sect. Lophogalium, sect. Platygalium, sect. Leiogalium, sect. Trachygalium, sect. Relbunium, sect. Depauperata, sect. Aparinoides. The members of sect. Depauperata, and sect. Aparinoides are sister to the rest of the species within this Clade. G. trifidum L. is paraphyletic and unresolved within the clade. Group A in Clade III included four taxa from $G$. trachygalium group $(G$. gracilens (A. Gray) Makino, G. koreanum, G. pogonanthum Franch. \& Sav. and $G$. trachyspermum A. Gray) and members of sect. Platygalium (BS: $80 \%$, PP $<0.95$ ). The previous classification based on morphological study of the four taxa of the G. trachygalium group was not resolved (Jeong and Pak, 2009). The individuals from the same taxa did not even form the monophyletic. G. kinuta Nakai \& Hara belonging to sect. Platygalium with G. boreale L. was resolved as paraphyletic. 


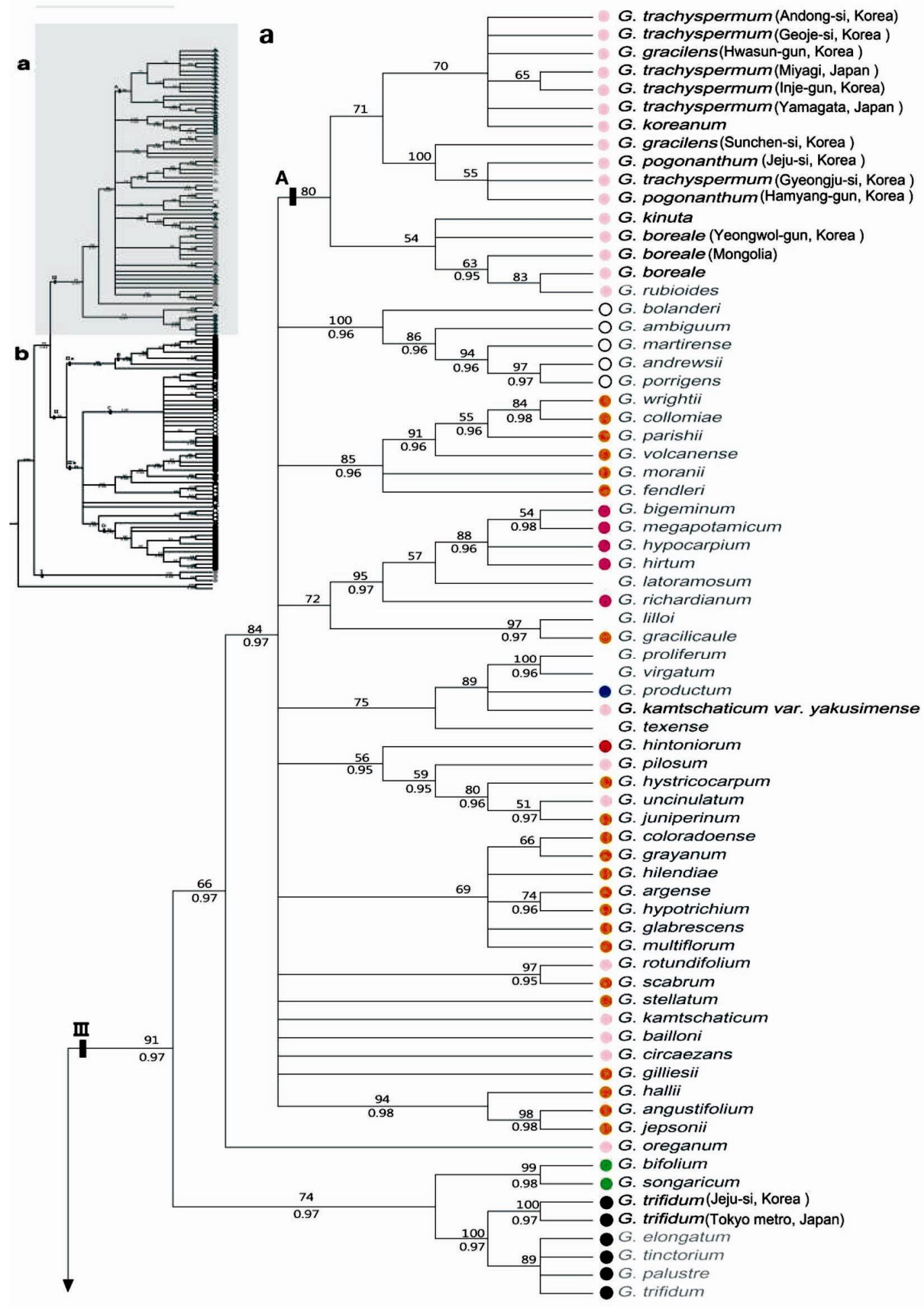




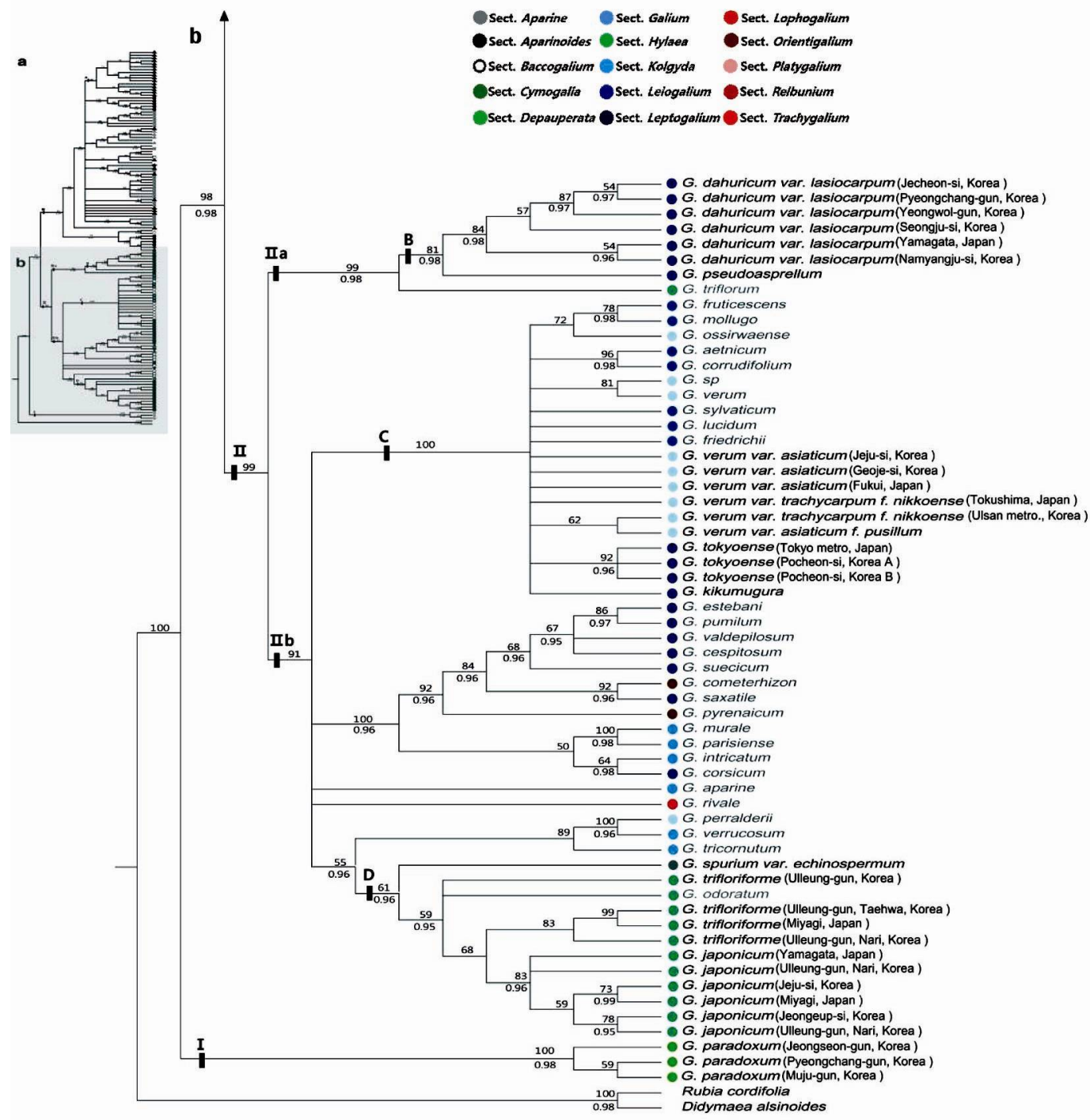

Fig. 1. Strict consensus tree of genus Galium based on Chloroplast DNA data, Bootstrap values and posterior probabilities are shown above and below branches, respectively. Different shapes were used for sectional treatments (taxon without shape "represents not classified"). Species in black represent the taxa sampled in this study.

\section{Phylogenetic relationships of Korean-Japanese Galium}

The phylogenetic relationships among Korean Galium and some of Japanese Galium were, for the first time, assessed in this study. We confirmed that the cpDNA phylogeny has significantly higher resolution and better support than previous study in Korean-Japanese Galium using morphological and chromosome number data by Jeong and Pak $(2009,2012)$. In some of taxa, our data were incongruent with previous classifications of Korean-Japanese Galium based on morphological data. 
G. paradoxum was sister to the group consisting of the rest of the Galium species (Fig 1). It also support the study of Ehrendorfer et al. (2014) using the plastid DNA sequences. The species is a perennial herb with opposite leaves, a pair of scale-like small stipules, one vein, white petiole and corolla, and rotate flowers. G. paradoxum was placed into a sect. Cymogalia based on the characters of inflorescence and hairs of a fruit (Pobedimova et al., 2000; Ehrendorfer et al., 2005). Its main distributions is in eastern Asia (Ehrendorfer et al., 2014), and mainly occurs in moist high elevations in mountain forests.

The taxa in the clade II have whorls of six or eight leaf-like organs. The five taxa from KoreaJapan are contained in Group C. The taxa of G.verum group (sect. Galium; G. verum var. asiaticum Nakai, G. verum f. nikkoense var. trachycarpum (Nakai) Ohwi and G. verum var. asiaticum f. pusillum) showed polytomies in the MPtree with weak PP. G. verum var. asiaticum is widely distributed throughout Korea and Japan. In our study, G. verum var. asiaticum have five chloroplast types from five individuals. But we cannot find morphological variation among the individuals. The three taxa are erect and have whorls of six or more than leaf-like organs, inflorescences of branched panicles with white or yellow flowers, and glabrous fruits. These three taxa don't exhibit significant morphological differences. But the plant and leaves size of G. verum var. asiaticum f. pusillum are smaller than those of other two taxa, and Korean endemic species in Mt. Halla on Jeju Island (Lee, 2004). It formed a clade with G. verum f. nikkoense var. trachycarpum from Ullsan-si (eastern part of Korea) with weak BS. It could provide crucial information for origin of Korean endemic, G. verum var. asiaticum $\mathrm{f}$. pusillum. It needs additional study to investigate the origin and in these evolutionary relationship among these taxa. The four taxa of $G$. dahuricum group from Korea-Japan; G. dahuricum var. lasiocarpum, $G$. kikumugura, G. tokyoense, and G. pseudoasprellum, are have been included into sect. Trachygalium (Ehrendorfer et al., 2005). There is no study of phylogenetic using molecular makers before. The four taxa of G. dahuricum group occur in East Asia, and have serious identification problems and taxon delimitation due to severe variations in the morphology of leaves, seed hairs and flower and inflorescences (Chen and Enrendorfer, 2011). We confirmed the phylogenetic relationship among these taxa, for the first time. G. kikumugura and G. tokyoense were included in Group C. G. kikumugura having whorls of four leaf-like organs and fruit with generally hooked hairs were closely related to G. tokyoense, morphologically (Yamazaki, 1993). Lee (1995) reported the distribution of G. kikumugura in Korea but we could not find the distibution during the this study although the species is widely distributed in Japan. We also could not confirm G. kikumugura specimens collected from Korea at Korean and Japan herbria. Therefore we assumed that the distribution report of this taxa by Lee (1995) was based on misclassification. G. pseudoasprellum was treated as synonyms of G. dahuricum by Ehrendorfer et al. (2005), but in our results did not support his opinion. G. pseudoasprellum is similar to $G$. dahuricum var. lasiocarpum, morphologically but it can be distinguished from G. dahuricum based on leaf shapes, which whorl of 6 elliptic or lanceolate leaves. G. tokyoense has glabrous fruit and white flower compare with G. dahuricum var. lasiocarpum. Previous studies based on morphology (Yamazaki, 1993; Pobedimova et al., 2000; Chen and Ehrendorfer, 2011) were argument for classification of G. tokyoense. We confirmed that the G. tokyoense and G. dahuricum var. lasiocarpum were polyphyletic. Also our result is supported that previous classification that G. tokyoense be regarded as a species. G. kamchaticum Steller ex Schultes \& J. H. Schultes and G. kamchaticum var. yakusimense (Masamune) Yamazakiwere place to clade $\square$ with polytomy at MP tree with weak PP value. G .kamchaticum is distributed in an alpine meadow of worldwide with centers of the diversity in eastern Asia and eastern North America (Ehrendorfer et al., 2005). G. kamchaticum var. yakusimense is smaller leave and tall than G. kamchaticum. This species is 
erect, with round leaves, one vein, whorls of four leaf-like organs, 4-parted white, and a fruit with generally hooked hairs.

We confirmed that $G$. kinuta is closer to $G$. boreale. Two taxa usually occur in northern part of Korean peninsula, especially in the mountain forests in lower elevation. The somatic chromosome number of $G$. kinuta and $G$. boreale were $4 \mathrm{X}(2 n=44)$ and/or $2 \mathrm{X}(2 n=11)$, respectively (Jeong and Pak, 2009). G. kinuta is erect, four leaf-like organs, three veins, branched panicles of inflorescences, and white flowers. G. kinuta and G. boreale are generally very similar in morphology and can be distinguished by the characters of leaf-shape.

Table 2. Primers used for amplification of cpDNA regions in this study.

\begin{tabular}{|c|c|c|c|c|}
\hline Region & Primer & Sequence $\left(5^{\prime}-3^{\prime}\right)$ & $\begin{array}{c}\text { Annealing } \\
\text { temperature }\left({ }^{\circ} \mathrm{C}\right)\end{array}$ & References \\
\hline \multirow[t]{2}{*}{$\operatorname{trn} \mathrm{C}-y c f 6$} & $\operatorname{trnC} C^{\mathrm{GCA}} \mathrm{F}$ & CCAGTTCRAATCYGGGTG & \multirow[t]{2}{*}{ ב } & Demesure et al. (1995) \\
\hline & ycf6R & GCCCAAGCRAGACTTACTATATCCAT & & Demesure et al. (1995) \\
\hline \multirow[t]{2}{*}{$\operatorname{trn} \mathrm{F}-n d h \mathrm{~J}$} & ndhJ & ATGCCYGAAAGTTGGATAGG & \multirow{2}{*}{57} & Shaw et al. (2007) \\
\hline & TabE & GGTTCAAGTCCСТCTATCCC & & Taberlet et al. (1991) \\
\hline \multirow[t]{2}{*}{$\operatorname{Trn} \mathrm{L}$ intron } & c & CGAAATCGGTAGACGCTACG & \multirow{2}{*}{55} & Taberlet et al. (1991) \\
\hline & $\mathrm{d}$ & GGGGATAGAGGGACTTGAAC & & Taberlet et al. (1991) \\
\hline \multirow[t]{2}{*}{ rpoB-trnC } & $\mathrm{rpoBb}$ & CGGATATTAATAKMTACATACG & \multirow{2}{*}{55} & Soza and Olmstead (2010) \\
\hline & rpoBd & GTTGGGGTTTACATATACT & & Soza and Olmstead (2010) \\
\hline
\end{tabular}

The G. trachygalium group consisted of four species; G. trachygalium, G. pogonanthum, G. gracilens, which occur in both Korea and Japan, and G. koreanum endemic to Korea. Although, the four taxa placed into Group A, our data did not provide insights into the specific phylogenetic relationships among G. trachygalium group species. These taxa are characterized by whorls of four leaf-like organs, cymose inflorescences with several terminal flowers, 4-parted rotate flowers and tuberculate fruit. The identification and delimitation of these species are usually difficult because they are very similar in morphology. The four species are distinguished by the differences in leaf size, shape, and fruit hairs (Jeong and Pak, 2012). These taxa usually occur in the near or same population, and share a common habitat. The somatic chromosome number of these species are $2 \mathrm{X}(2 n=22)$ and/or $4 \mathrm{X}(2 n=44)$ (Jeong and Pak, 2009). This inconsistencies phylogeny can be explained the speciation processes of the G. trachygaliumgroup. But it is yet to be determined whether incomplete lineage sorting of ancestral polymorphisms in the population, or chloroplast capture by hybridization and introgression. It needs additional study to understand origin and clear relationship among these taxa. G. trachyspermum, G. pogonanthum and G. gracilens previously been placed into a sect. Cymogalia by Yamazaki (1993) but our data showed that these four taxa including G. koreanum, are more closely related to members of sect. Platygalium (Table 1). We suggest that the four taxa have to be transferred to sect. Platygalium based on molecular and morphological data.

\section{Acknowledgement}

This research was supported by Basic Science Research Program through the National Research Foundation of Korea (NRF) funded by the Ministry of Education (2016R1A 6A1A05011910).

\section{References}

Chen, T. and Ehrendorfer, F. 2011. Rubia.Vol 19. In: Wu ZY, Raven PH, Hong DY(Eds). Flora of china. Beijing: Science Press; St. Louis: Missouri Botanical Garden Press. pp. 104-141. 
Demesure, B., Sodzi, N., Petit, R.J. 1995. A set of universal primers for amplification of polymorphic noncoding regions of mitochondrial and chloroplast DNA in plants. Molec. Ecol. 4: 129-131.

Dolye, J.J. and Dolye, J.L.1987. A rapid DNA isolation procedure for small quantities of fresh leaf tissue.Phytochem. Bul. Bot. Soc. Amer. 19:11-15.

Ehrendorfer, F., Manen, J.-F.and Natali, A. 1994. Cp DNA intergene sequences corroborate restriction site data for reconstructing Rubiaceae phylogeny. Pl. Syst. Evol. 190: 195-211.

Ehrendorfer, F., Schönbeck-Temesy, E., Puff, C. and Rechinger, W. 2005. Rubiaceae.eds. K. H. Rechinger, Flora Iranica. no. 176. Verlag des Naturhistorischen Museums Wien, Vienna, Austria.

Ehrendorfer, F., Vladimirov, V. and Barfuss, M.H.J. 2014. Paraphyly and polyphyly in the worldwide tribe Rubieae (Rubiaceae): Challenges for genetic delimitation. Ann. Missouri Bot. Gard. 100: 79-88.

Felsensteijn, J. 1985. Confidence limits on phylogenies: an approach using the bootstrap. Evolution. 39: 783791.

Govaerts, R. 2006. World checklist of selected plant families. In: F. A. Bisby, Y.R. Roskov, M. A. Ruggiero, T. M. Orrell, L. E. Paglinawan, P. W. Brewer, N.Bailly, and J. van Hertum, (eds.), Species 2000 \& ITIS catalogue of life: www.catalogueoflife.org/annualchecklist/ 2007/. Species 2000, Reading, U. K.

Jeong K.S. and Pak, J. H. 2009. A cytotaxonomic study of Galium (Rubiaceae) in Korea. Korean J. Pl. Tax. 39: 42-47.

Jeong K.S. and Pak, J. H. 2012. The morphological study of Galium L. (Rubiaceae) in Korea. Korean J. Pl. Tax. 42: 1-12.

Lee, T.B. 1979. Illustrated Flora of Korea. Hyangmunsa, Seoul (in Korean).

Lee, W.T. 1995. Lineamenta Florae Koreae. Academy Press, Seoul (in Korean).

Lee, Y.N. 2004. Flora of Korea. Kyohaksa, Seoul (in Korean).

Manen, J.-F., Natali, A. and Ehrendorfer, F. 1994. Phylogeny of Rubiaceae-Rubieae inferred from the sequence of a cpDNA intergene region.Pl. Syst. Evol.190: 195-211.

Manen, J.-F.and Natali, A. 1995. Comparison of the evolution of ribulose-1,5-biphosphate carboxylase $(r b c L)$ and $a t b-r b c L$ noncoding spacer sequences in a recent plant group, the tribe Rubieae (Rubiaceae). J. Molec. Evol. 41: 920-927.

Natali, A., Manen, J.-F.andEhrendorfer, F. 1995. Phylogeny of the Rubiaceae-Rubioideae, in particular the tribe Rubieae: Evidence from a non-coding chloroplast DNA sequence. Ann. Missouri Bot. Gard. 82: 428-439.

Natali, A., Manen, J.-F., Kiehn, M. and Ehrendorfer, F. 1996. Tribal, generic, and specific relationships in the Rubioideae-Rubieae (Rubiaceae) based on sequence data of a cpDNA intergene region. Opera Bot. Belg. 7: 193-203.

Nylander, J.A.A. 2004. Mrmodeltest 2.3. Program distributed by the author. Evolutionary biology Centre, Uppsala University.

Pobedimova, E.G. 2000. Galium L. in: B. K. Schischkin (ed.), Flora of the U.S.S.R, vol. 23. Bishen Singh Mahendra Pal Singh, Dehra Dun. India, and Koeltz Scientific Books, Koenigstein, Germany. pp. 345459.

Robbrecht, E. and Manen, J.F. 2006. The major evolutionary lineages of the coffee family (Rubiaceae, angiosperms). Combined analysis (nDNA and cpDNA) to infer the position of Coptosapelta and Luculia, and supertree construction based on $r b c \mathrm{~L}, r p s 16$, $\operatorname{trn} \mathrm{L}-\operatorname{trn} \mathrm{F}$ and $a t p \mathrm{~B}-r b c \mathrm{~L}$ data. A new classification in two subfamilies, Cinchonoideae and Rubioideae. Syst. Geogr. Plants. 76: 85-146.

Ronquist, F. and Huelsenbeck, J.P. 2003. Mrbayes 3: Bayesian phylogenetic inference under mixed models. Bioinfomatics. 19: 1572-1574.

Shaw, J., E. B. Lickey, J. T. Beck, S. B. Farmer, W. Liu, J. Miller, K. C. Siripun, C. T. Winder, E. E. Schilling, and R. L. Small. 2005. The tortoise and the hare II: Relative utility of 21 noncoding chloroplast DNA sequences for phylogenetic analysis. Am. J. Bot. 92: 142-166.

Shaw, J., Lickey, E.B., Schililling, E.E. and Small, R.L. 2007. Comparison of whole chloroplast genome sequences to choose noncoding regions for phylogenetic studies in angiosperms; the tortoise and the hare. Am. J. Bot. 94: 275-288. 
Soza, V.L. and Olmstead, R.G. 2010. Molecular systematics of tribe Rubieae (Rubiaceae): Evolution of major clades, development of leaf-like whorls, and biogeography. Taxon 59: 755-771.

Swofford, D.L. 2003. PAUP: Phylogenetic analysis using parsimony (and other method). Ver.4.0b10. Sinauer Associates, Sunderlad, Massachusetts.

Taberlet, P., Gielly, L. Pautou, G. and Bouvet, J.1991. Universal primer for amplification of three non-coding regions of chloroplast DNA.Pl.Molec. Biol. 17: 1105-1109.

Thompson, J.D., Gibson, T.J., Plewniak, F., Jeanmougin, F. and Higins, D.G. 1997. The CLUSTAL X windows interface: flexible strategies for multiple sequence alignment aided by quality analysis tools. Nucleic Acids Res. 22: 4676-4882.

Yamazaki, T. 1993. Angiospermae. Vol $\square$ a. In: Iwatsuki K., David E.B., Ohba H. (Eds.) Flora of Japan. Tokyo: Kodansa. pp. 233-240.

(Manuscript received on 2 October 2015; revised on 1 November 2016) 